\title{
Approximation of derivations and the superstability in random Banach $*$-algebras
}

\section{Reza Saadati ${ }^{*}$ (ID and Choonkil Park ${ }^{2}$}

\section{"Correspondence:}

rsaadati@iust.ac.ir; rsaadati@eml.cc ${ }^{1}$ Department of Mathematics, Iran University of Science and Technology, Tehran, Iran Full list of author information is available at the end of the article

\begin{abstract}
We prove that approximations of derivations on random Banach *-algebras are exactly derivations by using a fixed point method. Furthermore, we show that approximations of quadratic $*$-derivations on random Banach $*$-algebras are exactly quadratic $*$-derivations. We, moreover, prove that approximations of derivations on random $C^{*}$-ternary algebras are exactly derivations by using a fixed point method.

MSC: 46S50; 47H10; 26E60

Keywords: Derivation; Quadratic derivation; Superstability; Fixed point method; Random Banach *-algebra; Random C*-ternary algebra
\end{abstract}

\section{Introduction}

Ulam [1] presented an effective lecture at the University of Wisconsin in which he stated a number of essential unsolved problems, in the fall of 1940. The next question concerning the stability of homomorphisms was among those:

Assume that $\Omega_{1}$ is a group and suppose that $\Omega_{2}$ is a metric group with a metric $\Delta(\cdot, \cdot)$. Let $\xi>0$, is there $\eta>0$ such that if a function $\varphi: \Omega_{1} \rightarrow \Omega_{2}$ satisfies the inequality $\Delta(\varphi(u v), \varphi(u) \varphi(v))<\eta$ for all $u, v \in \Omega_{1}$ then there is a homomorphism $\Phi: \Omega_{1} \rightarrow \Omega_{2}$ with $\Delta(\varphi(u), \Phi(u))<\xi$ for all $u \in \Omega_{1}$ ?

When the answer is established, the functional equation for homomorphisms is stable.

The first mathematician who presented the result concerning the stability of functional equations was Hyers [2]. He intelligently answered Ulam's question when $\Omega_{1}$ and $\Omega_{2}$ are Banach spaces. Recently, Rassias [3] and others have obtained important results on stability and applied them to the investigations in the nonlinear sciences.

\section{Preliminaries}

Assume that $\Delta^{+}$is the family of distribution functions, i.e., the family of all left-continuous functions $G:[-\infty, \infty] \rightarrow[0,1]$ such that $G$ is increasing on $[-\infty, \infty], G(0)=0$ and $G(+\infty)=1 . D^{+} \subseteq \Delta^{+}$contains each function $G \in \Delta^{+}$for which $\ell^{-} G(+\infty)=1$ and $\ell^{-} g(x)$ is the left limit of the map $g$ at $x$, i.e., $\ell^{-} g(x)=\lim _{t \rightarrow x^{-}} g(t)$. In $\Delta^{+}$, we have $H \leq F$ if and only if $H(s) \leq F(s)$ for all $s$ in $\mathbb{R}$ (partially ordered). Note that the function $\varepsilon_{u}$ defined by

$$
\varepsilon_{u}(s)= \begin{cases}0, & \text { if } s \leq u, \\ 1, & \text { if } s>u,\end{cases}
$$

(c) The Author(s) 2018. This article is distributed under the terms of the Creative Commons Attribution 4.0 International License (http://creativecommons.org/licenses/by/4.0/), which permits unrestricted use, distribution, and reproduction in any medium, provided you give appropriate credit to the original author(s) and the source, provide a link to the Creative Commons license, and indicate if changes were made. 
is an element of $\Delta^{+}$and $\varepsilon_{0}$ is the maximal element in this space. For more details see [4-6].

Definition 2.1 ([6]) Let $I=[0,1]$. A continuous triangular norm (briefly, $c t$-norm) is a function $T$ from $I$ to $I$ with continuity property such that:

(a) $T(\theta, \vartheta)=T(\vartheta, \theta)$ and $T(\theta, T(\vartheta, \iota))=T(T(\theta, \vartheta), \iota)$ for all $\theta, \vartheta, \iota \in I$;

(b) $T(\theta, 1)=\theta$ for $0 \leq \theta \leq 1$;

(c) $T(\theta, \vartheta) \leq T(\iota, \kappa)$ whenever $\theta \leq \iota$ and $\vartheta \leq \kappa$ for each $\theta, \vartheta, \iota, \kappa \in I$.

$T_{P}(\theta, \vartheta)=\theta \vartheta, T_{M}(\theta, \vartheta)=\min (\theta, \vartheta)$ and $T_{L}(\theta, \vartheta)=\max (\theta+\vartheta-1,0)$ (the Lukasiewicz $t$-norm) are some examples of $t$-norms. Also, we define $\prod_{j=1}^{n} \theta_{j}=T^{n-1}\left(\theta_{1}, \ldots, \theta_{n}\right)$.

Definition 2.2 ([6]) Suppose that $T$ is a $c t$-norm, $V$ is a vector space and let $\mu$ be a map from $V$ to $D^{+}$. In this case, the ordered triple $(V, \mu, T)$ with the properties

(RN1) $\mu_{v}(\theta)=\varepsilon_{0}(\theta)$ for all $\theta>0$ if and only if $v=0$

(RN2) $\mu_{\alpha \nu}(\theta)=\mu_{\nu}\left(\frac{\theta}{|\alpha|}\right)$ for all $v \in V, \alpha \neq 0$;

(RN3) $\mu_{u+v}(\theta+\vartheta) \geq T\left(\mu_{u}(\theta), \mu_{v}(\vartheta)\right)$ for all $u, v \in V$ and all $\theta, \vartheta \geq 0$,

is said to be a random normed space (in short, $\mathrm{RN}$-space).

Let $(V,\|\cdot\|)$ be a linear normed space. Then

$$
\mu_{\nu}(\vartheta)=\frac{\vartheta}{\vartheta+\|v\|}
$$

for all $\vartheta>0$, defines a random norm, and the ordered triple $\left(V, \mu, T_{M}\right)$ is an RN-space.

Definition 2.3 Assume that the following algebraic structure on an $\mathrm{RN}$-space $(V, \mu, T)$ holds:

(RN-4) $\mu_{u v}(\theta \vartheta) \geq T^{\prime}\left(\mu_{u}(\theta), \mu_{v}(\vartheta)\right)$ for each $u, v \in V$ and all $\theta, \vartheta>0$, where $T^{\prime}$ is a $c t$-norm.

Then $\left(V, \mu, T, T^{\prime}\right)$ is called a random normed algebra.

Suppose that $(V,\|\cdot\|)$ is a normed algebra. Then $\left(V, \mu, T_{M}, T_{P}\right)$ is a random normed algebra, where

$$
\mu_{v}(\vartheta)=\frac{\vartheta}{\vartheta+\|v\|}
$$

for all $\vartheta>0$ if and only if

$$
\|u v\| \leq\|v\|\|u\|+\theta\|u\|+\vartheta\|v\| \quad(v, u \in V ; \theta, \vartheta>0) .
$$

For more details, see [7-22].

Definition 2.4 A random Banach $*$-algebra $\mathcal{B}$ is a random complex Banach algebra $\left(\mathcal{B}, \mu, T, T^{\prime}\right)$, together with an involution on $\mathcal{B}$ which is a mapping $g \mapsto g^{*}$ from $\mathcal{B}$ into $\mathcal{B}$ that satisfies

(i) $g^{* *}=g$ for $g \in \mathcal{B}$; 
(ii) $(a g+b h)^{*}=\bar{a} g^{*}+\bar{b} h^{*}$;

(iii) $(g h)^{*}=h^{*} g^{*}$ for $g, h \in \mathcal{B}$.

If, in addition, $\mu_{g^{*} g}(\theta \vartheta)=T^{\prime}\left(\mu_{g}(\theta), \mu_{g}(\vartheta)\right)$ for $g \in \mathcal{B}$ and $\theta, \vartheta>0$, then $\mathcal{B}$ is called a random $C^{*}$-algebra.

Assume that $\mathcal{B}$ is a random Banach $*$-algebra. A derivation on $\mathcal{B}$ is a mapping $\delta$ from $\mathcal{B}$ to $\mathcal{B}$ such that:

$$
\begin{aligned}
& \delta(\lambda g+h)=\lambda \delta(g)+\delta(h), \\
& \delta(g h)=\delta(g) h+g \delta(h)
\end{aligned}
$$

for all $g, h \in \mathcal{B}$ and all $\lambda \in \mathbb{C}$. A derivation $\delta$ is called a $*$-derivation on $\mathcal{B}$ if $\delta\left(g^{*}\right)=\delta(g)^{*}$ for all $g \in \mathcal{B}$ (see [23]).

Recall that

$$
\begin{aligned}
& \omega(u+v)=\omega(u)+\omega(v), \\
& \omega(u+v)+\omega(u-v)=2 \omega(u)+2 \omega(v),
\end{aligned}
$$

respectively, are Cauchy additive and Cauchy quadratic functional equations.

Firstly, Baker, Lawrence and Zorzitto [24] defined the concept of superstability. Let $\left(\mathcal{B}, \mu, T, T^{\prime}\right)$ be an RN algebra. The random norm is multiplicative if $\mu_{u v}(\theta \vartheta)=T^{\prime}\left(\mu_{u}(\theta)\right.$, $\left.\mu_{v}(\vartheta)\right)$ for all $u, v \in \mathcal{B}$ and all $\theta, \vartheta>0$.

Suppose that $\Gamma \neq \emptyset$. A function $\Delta: \Gamma \times \Gamma \rightarrow[0, \infty]$ is a generalized metric (GM) on $\Gamma$ if

(1) $\Delta(\rho, \varrho)=0$ if and only if $\rho=\varrho$;

(2) $\Delta(\rho, \varrho)=\Delta(\varrho, \rho)$ for all $\rho, \varrho \in \Gamma$;

(3) $\Delta(\rho, \varrho) \leq \Delta(\rho, \sigma)+\Delta(\sigma, \varrho)$ for all $\rho, \varrho, \sigma \in \Gamma$.

Theorem $2.1([25,26])$ Suppose that $(\Gamma, \Delta)$ is a complete GM space and assume that the selfmapping $\Upsilon$ on $\Gamma$ with Lipschitz constant $0<L<1$ is strictly contractive. Then, for $\varrho \in \Gamma$, either

$$
\Delta\left(\Upsilon^{n} \varrho, \Upsilon^{n+1} \varrho\right)=\infty
$$

for each $0 \leq n \in \mathcal{Z}$, or there exists $n_{0} \in \mathbb{N}$ such that

(1) $\Delta\left(\Upsilon^{n} \varrho, \Upsilon^{n+1} \varrho\right)<\infty, \forall n \geq n_{0}$;

(2) the sequence $\left\{\Upsilon^{n} \varrho\right\}$ tends to $\sigma^{*}$ in $\Gamma$;

(3) $\Upsilon\left(\sigma^{*}\right)=\sigma^{*}$;

(4) $\Upsilon\left(\sigma^{*}\right)=\sigma^{*}$ and is unique in $\mathbb{E}=\left\{\sigma \in \Gamma \mid \Delta\left(\Upsilon^{n_{0}} \varrho, \sigma\right)<\infty\right\}$

(5) $(1-L) \Delta\left(\sigma, \sigma^{*}\right) \leq \Delta(\sigma, \Upsilon \sigma)$ for all $\sigma \in \Gamma$.

\section{Approximation of derivations on random Banach $*$-algebras}

Assume that a random $*$-Banach algebra $\mathcal{B}$ has unit $e$. Our results improve and expand the result presented by Jang [27]. 
Theorem 3.1 Let $\psi_{1}: \mathcal{B} \times \mathcal{B} \rightarrow D^{+}$and $\psi_{2}: \mathcal{B} \rightarrow D^{+}$be distribution functions. Assume that $: \mathcal{B} \rightarrow \mathcal{B}$ is a mapping such that

$$
\begin{aligned}
& \mu_{f(\xi p+q)-\xi f(p)-f(q)}(t) \geq \psi_{1}(p, q, t), \\
& \mu_{f(p q)-p f(q)-f(p) q}(t) \geq \psi_{1}(p, q, t), \\
& \mu_{f\left(p^{*}\right)-f(p)^{*}}(t) \geq \psi_{2}(p, t),
\end{aligned}
$$

for all $\xi \in \mathbb{T}, p, q \in \mathcal{B}$ and $t>0$. If there exist $n \in \mathbb{N}$ and $0<L<1$ such that $\psi_{1}(s p, s q, L s t)>$ $\psi_{1}(p, q, t), \psi_{1}(s p, q, L s t)>\psi_{1}(p, q, t), \psi_{1}(p, s q, L s t)>\psi_{1}(p, q, t)$ and $\psi_{2}(s p, L s t)>\psi_{2}(p, t)$ for all $p, q \in \mathcal{B}$ and $t>0$. Then $f$ on $\mathcal{B}$ is $a *$-derivation.

Proof Putting $p=q$ and $\xi=1$ in (3.1), we get

$$
\mu_{f(2 p)-2 f(p)}(t) \geq \psi_{1}(p, p, t)
$$

for all $p \in \mathcal{B}$ and $t>0$. By induction, we can prove that

$$
\mu_{f(n p)-n f(p)}(t) \geq \prod_{j=1}^{n-1} \psi_{1}\left(j p, p, t_{j}\right)
$$

for all $p, q \in \mathcal{B}, t>0$ and $n \geq 2$ where $\sum_{j=1}^{n-1} t_{j}=t$.

Define

$$
\Psi(p, t)=\prod_{j=1}^{s-1} \psi_{1}\left(j p, p, t_{j}\right)
$$

for $p \in \mathcal{B}, t>0$ and $s \geq 2$ where $\sum_{j=1}^{s-1} t_{j}=t$. So

$$
\mu_{f(s p)-s f(p)}(t) \geq \Psi(p, t)
$$

Put $\Gamma=\{g ; g: \mathcal{B} \rightarrow \mathcal{B}\}$. Define a function $\Delta: \Gamma \times \Gamma \rightarrow[0, \infty]$ such that

$$
\Delta(\vartheta, v)=\inf \left\{v>0: \mu_{\vartheta(p)-v(p)}(v t) \geq \Psi(p, t), \forall p \in \mathcal{B}, t>0\right\}
$$

where $\vartheta, v \in \Gamma$. Miheț and Radu [28] proved that $(\Gamma, \Delta)$ is a complete GM space. Define a mapping $H: \Gamma \rightarrow \Gamma$ by $H(\vartheta)(p)=s^{-1} v(s p)$. Put

$$
\Delta(\vartheta, v)=v,
$$

where $\vartheta, v \in \Gamma$. Then

$$
\mu_{H(\vartheta)(p)-H(v)(p)}(t)=\mu_{\vartheta(s p)-v(s p)}(s t) \geq \Psi\left(s p, \frac{s}{\alpha} t\right) \geq \Psi\left(p, \frac{t}{L \alpha}\right) .
$$

So, for $\vartheta, v \in S$, we have

$$
\Delta(H(\vartheta), H(v)) \leq L \Delta(\vartheta, v) .
$$


Then the mapping $H$ on $\Gamma$ with Lipschitz constant $L$ is strictly contractive. From (3.6), we have

$$
\mu_{(H f)(p)-f(p)}(t)=\mu_{f(s p)-f(p)}(s t)=\mu_{f(s p)-s f(p)}(s t) \geq \Psi(p, s t)
$$

which implies that $\Delta(H(f), f) \leq 1 /|s|$. Theorem 2.1 implies that, in the set

$$
U=\{\vartheta \in \Gamma: \Delta(\vartheta, H(f))<\infty\}
$$

$h: \mathcal{B} \rightarrow \mathcal{B}$ is a unique fixed point of $H$. Also for every $p \in \mathcal{A}$

$$
h(p)=\lim _{m \rightarrow \infty} H^{m}(f(p))=\lim _{m \rightarrow \infty} s^{-m} f\left(s^{m} p\right) .
$$

Using (3.6), we get

$$
\begin{aligned}
\mu_{h(\xi p+q)-\xi h(p)-h(q)}(t) & =\lim _{n \rightarrow \infty} \mu_{f\left(s^{n}(\xi p+q)\right)-\xi f\left(s^{n} p\right)-f\left(s^{n} q\right)}\left(s^{n} t\right) \\
& \geq \lim _{n \rightarrow \infty} \psi_{1}\left(s^{n} p, s^{n} q, s^{n} t\right) \\
& \geq \lim _{n \rightarrow \infty} \psi_{1}\left(p, q, \frac{t}{L^{n}}\right)=1
\end{aligned}
$$

for all $p, q \in \mathcal{B}, \xi \in T$ and $t>0$. Let $\xi=\xi_{1}+i \xi_{2} \in \mathbb{C}, \xi_{1}, \xi_{2} \in \mathbb{R}$ and let $\mu_{1}=\xi_{1}-\left[\xi_{1}\right]$ and $\mu_{2}=\xi_{2}-\left[\xi_{2}\right]$ where $[\xi]$ denotes the integer part of $\xi$. So $0 \leq \mu_{i}<1(1 \leq i \leq 2)$. Now, we represent $\mu_{i}$ as $\mu_{i}=\frac{\xi_{i, 1}+\xi_{i, 2}}{2}$ such that $\xi_{i, j} \in \mathbb{T}(1 \leq i, j \leq 2)$. Since $h(\xi p+q)=\lambda h(p)+h(q)$ for $\xi \in T$, we conclude that

$$
\begin{aligned}
h(\xi p) & =h\left(\xi_{1} p\right)+i h\left(\xi_{2} p\right) \\
& =\left(\left[\xi_{1}\right] h(p)+\delta\left(\mu_{1} p\right)\right)+i\left(\left[\xi_{2}\right] h(p)+h\left(\mu_{2} p\right)\right) \\
& =\left(\left[\xi_{1}\right] h(p)+\frac{1}{2} h\left(\xi_{1,1} p+\xi_{1,2} p\right)\right)+i\left(\left[\xi_{2}\right] h(p)+\frac{1}{2} h\left(\xi_{2,1} p+\xi_{2,2} p\right)\right) \\
& =\left(\left[\xi_{1}\right] h(p)+\frac{1}{2} \xi_{1,1} h(p)+\frac{1}{2} \xi_{1,2} h(p)\right)+i\left(\left[\xi_{2}\right] h(p)+\frac{1}{2} \xi_{2,1} h(p)+\frac{1}{2} \xi_{2,2} h(p)\right) \\
& =\xi_{1} h(p)+i \xi_{2} h(p) \\
& =h(p)
\end{aligned}
$$

for all $p \in \mathcal{B}$ and $\xi \in \mathbb{C}$. So, on $\mathcal{B}, h$ is a $\mathbb{C}$-linear mapping. For the involution of $h$, we have

$$
\begin{aligned}
\mu_{h\left(p^{*}\right)-h(p)^{*}}(t) & =\lim _{n \rightarrow \infty} \mu_{f\left(s^{n} p^{*}\right)-f\left(s^{n} p\right)^{*}}\left(s^{n} t\right) \\
& \geq \lim _{n \rightarrow \infty} \psi_{2}\left(s^{n} p, s^{n} t\right) \\
& \geq \lim _{n \rightarrow \infty} \psi_{2}\left(p, \frac{t}{L^{n}}\right) \\
& =1 .
\end{aligned}
$$


Now, we prove the derivation property of $h$. In (3.2), we replace $p$ by $s^{n} p, q$ by $s^{n} q$, divide by $s^{2 n}$ and get

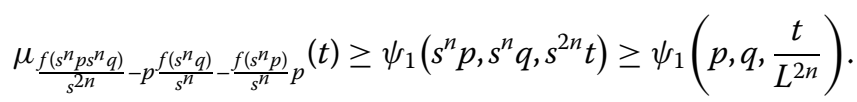

In (3.9), letting $n \rightarrow \infty$, we get

$$
h(p q)=p h(q)+h(p) q
$$

for all $p, q \in \mathcal{B}$. So $h$ is a $*$-derivation on $\mathcal{B}$. Now, in (3.2), replacing $p$ by $s^{n} p$ and dividing by $s^{n}$, we get

$$
\mu_{\frac{f\left(s^{n} p q\right)}{s^{n}}-p f(q)-\frac{f\left(s^{n} p\right)}{s^{n}} q}(t) \geq \psi_{1}\left(s^{n} p, q, s^{n} t\right) \geq \psi_{1}\left(p, q, \frac{t}{L^{n}}\right)
$$

for all $p, q \in \mathcal{B}, n \in \mathbb{N}$ and $t>0$. Letting $n \rightarrow \infty$, we get

$$
h(p q)=p f(q)+h(p) q
$$

for all $p, q \in \mathcal{B}$. Fix $m \in \mathbb{N}$. From

$$
\begin{aligned}
p f\left(s^{m} q\right) & =h\left(s^{m} p q\right)-h(p) s^{m} q \\
& =s^{m} p f(q)
\end{aligned}
$$

for all $p, q \in \mathcal{B}$, we have $p f(q)=p \frac{f\left(s^{m} q\right)}{s^{m}}$ for all $p, q \in \mathcal{B}$ and $m \in \mathbb{N}$. Letting $m \rightarrow \infty$, we get $p f(q)=p h(q)$. Putting $p=e$, we get $h(q)=f(q)$ for all $q \in \mathcal{B}$. Hence $f$ is a $*$-derivation on $\mathcal{B}$.

\section{Approximation of quadratic $*$-derivations on random Banach $*$-algebras}

Definition 4.1 Assume that a mapping $\delta: \mathcal{B} \rightarrow \mathcal{B}$ satisfies

(1) $\delta(\eta+\kappa)+\delta(\eta-\kappa)-2 \delta(\eta)-2 \delta(\kappa)=0$

(2) $\delta$ is quadratic homogeneous, that is, $\delta(\lambda \eta)=\lambda^{2} \delta(\eta)$;

(3) $\delta(\eta \kappa)=\delta(\eta) \kappa^{2}+\eta^{2} \delta(\kappa)$;

(4) $\delta\left(\eta^{*}\right)=\delta(\eta)^{*}$

for all $\eta, \kappa \in \mathcal{B}$ and $\lambda \in \mathbb{C}$. Then it is called a $*$-quadratic derivation on $\mathcal{B}$.

Theorem 4.2 Assume that $\psi_{1}: \mathcal{B} \times \mathcal{B} \rightarrow D^{+}$and $\psi_{2}: \mathcal{B} \rightarrow D^{+}$are distribution functions. Let $f: \mathcal{B} \rightarrow \mathcal{B}$ be a function such that

$$
\begin{aligned}
& \mu_{f(p+q)+f(p-q)-2 f(p)-2 f(q)}(t) \geq \psi_{1}(p, q, t), \\
& \mu_{f(p q)-p^{2} f(q)-f(p) q^{2}}(t) \geq \psi_{1}(p, q, t), \\
& \mu_{f(\xi p)-\lambda^{2} f(p)}(t) \geq \psi_{2}(p, t), \\
& \mu_{f\left(p^{*}\right)-f(p) *}(t) \geq \psi_{2}(p, t),
\end{aligned}
$$


for all $\xi \in \mathbb{C}, p, q \in \mathcal{B}$ and $t>0$. If there exist $s \in \mathbb{N}$ and $0<L<1$ such that $\psi_{1}\left(2^{s} p, 2^{s} q\right.$, $\left.2^{2 s} L t\right)>\psi_{1}(p, q, t), \psi_{1}\left(2^{s} p, q, 2^{2 s} L t\right)>\psi_{1}(p, q, t), \psi_{1}\left(p, 2^{s} q, 2^{2 s} L t\right)>\psi_{1}(p, q, t)$ and $\psi_{2}\left(2^{s} p\right.$, $\left.2^{2 s} L t\right)>\psi_{2}(p, t)$ for all $p, q \in \mathcal{B}$ and $t>0$. Then, on $\mathcal{B}, f$ is a $*$-quadratic derivation.

Proof Putting $p=q$ and $\xi=1$ in (4.1), we get

$$
\mu_{f(2 p)-4 f(p)}(t) \geq \psi_{1}(p, p, t)
$$

for all $p \in \mathcal{B}$ and $t>0$. Induction on $n$ yields

$$
\mu_{f\left(2^{n} p\right)-2^{2 n} f(p)}(t) \geq \prod_{i=0}^{n-1} \psi_{1}\left(2^{i} p, 2^{i} p, \frac{t_{i}}{2^{2(n-i)}}\right)
$$

for all $p, q \in \mathcal{B}, n \geq 2$ and $t>0$ where $\sum_{i=0}^{n-1} t_{i}=t$. Define

$$
\Psi(p, t)=\prod_{i=0}^{s-1} 2^{2(s-i)} \psi_{1}\left(2^{i} p, 2^{i} p, \frac{t_{i}}{2^{2(n-i)}}\right) .
$$

Then we have

$$
\mu_{f\left(2^{s} p\right)-2^{2 s} f(p)}(t) \geq \Psi(p, t) .
$$

The set of all mappings $\zeta: \mathcal{B} \rightarrow \mathcal{B}$ is denoted by $\Gamma$. Define a function $\Delta: \Gamma \times \Gamma \rightarrow[0, \infty]$ by

$$
\Delta(\zeta, \eta)=\inf \left\{v>0: \mu_{\zeta(p)-\eta(p)}(t) \geq \Psi\left(p, \frac{t}{v}\right), \forall p \in \mathcal{B}\right\}
$$

Miheț and Radu [28] proved that $(\Gamma, \Delta)$ is a complete GM space. Now, define a mapping $H: \Gamma \rightarrow \Gamma$ by $H(\zeta)(p)=2^{-2 s} \zeta\left(2^{s} p\right)$. Putting

$$
\Delta(\zeta, \eta)=v \quad(\zeta, \eta \in \Gamma)
$$

we obtain

$$
\mu_{H(\zeta)(p)-H(\eta)(p)}(t)=\mu_{\zeta\left(2^{s} p\right)-\eta\left(2^{s} p\right)}\left(\frac{t}{2^{2 s}}\right) \geq \Psi\left(2^{s} p, \frac{t}{v 2^{2 s}}\right) \geq \Psi\left(p, \frac{t}{L \alpha}\right) .
$$

Then, for $\zeta, \eta \in S$, we have

$$
\Delta(H(\zeta), H(\eta)) \leq L \Delta(\zeta, \eta)
$$

which means that $H$ on $\Gamma$, with Lipschitz constant $L$ is a strictly contractive mapping. Also, for $p \in \mathcal{B}$, we have

$$
\mu_{(H f)(p)-f(p)}(t)=\mu_{2^{-2 s} f\left(2^{s} p\right)-f(p)}(t)=\mu_{f\left(2^{s}\right) 2^{2 s} f(p)}\left(2^{2 s} t\right) \geq \Psi\left(p, 2^{2 s} t\right),
$$


which implies that $\Delta(H(f), f) \leq 1 / 2^{2 s}$. Using Theorem 2.1, we conclude that, in the set

$$
U=\{\zeta \in \Gamma: \Delta(\zeta, H(f))<\infty\}
$$

and for each $p \in \mathcal{B}, h: \mathcal{B} \rightarrow \mathcal{B}$ is a unique fixed point of $H$ and

$$
h(p)=\lim _{m \rightarrow \infty} H^{m}(f(p))=\lim 2^{-2 s m} f\left(2^{s m} p\right) .
$$

By (4.9), we have

$$
\begin{aligned}
& \mu_{h(p+q)+h(p-q)-2 h(p)-2 h(q)}(t) \\
& \quad=\lim _{n \rightarrow \infty} \mu_{f\left(2^{s n}(p+q)+f\left(2^{s n}(p-q)\right)-2 f\left(2^{s n} p\right)-2 f\left(2^{s n} q\right)\right.}\left(2^{2 s n} t\right) \\
& \geq \lim _{n \rightarrow \infty} \psi_{1}\left(2^{n s} p, 2^{n s} q, 2^{2 n s} t\right) \geq \lim _{n \rightarrow \infty} \psi_{1}\left(p, q, \frac{t}{L^{n}}\right)=1
\end{aligned}
$$

for all $p, q \in \mathcal{B}$ and $t>0$. Then $h$ is a quadratic mapping on $\mathcal{B}$. Also, we have

$$
\begin{aligned}
\mu_{h(\xi p)-\lambda^{2} h(p)}(t) & =\lim _{n \rightarrow \infty} \mu_{f\left(2^{n s}(\xi p)-\lambda^{2} f\left(2^{n s} p\right)\right.}\left(2^{2 n s} t\right) \\
& \geq \lim _{n \rightarrow \infty} \psi_{2}\left(2^{n s} p, 2^{2 n s} t\right) \\
& \geq \lim _{n \rightarrow \infty} \psi_{2}\left(p, \frac{t}{L^{n}}\right) \\
& =1,
\end{aligned}
$$

which implies that $h$ is quadratic homogeneous.

Now, replacing $p$ by $2^{n s} p$ in (4.2) and dividing by $2^{-2 s n}$, we get

$$
\mu_{\frac{f\left(2^{n s} p q\right)}{2^{2 n s}}-p^{2} f(q)-\frac{f\left(2^{n s} p\right)}{2^{2 n s}} q^{2}}(t) \geq \psi_{1}\left(2^{n s} p, q, 2^{2 n s} t\right) \geq \psi_{1}\left(p, q, \frac{t}{L^{n}}\right)
$$

for all $p, q \in \mathcal{B}, n \in \mathbb{N}$ and $t>0$. Letting $n \rightarrow \infty$, we get

$$
h(p q)=p^{2} f(q)+h(p) q^{2}
$$

for all $p, q \in \mathcal{B}$. Let $m \in \mathbb{N}$. We have

$$
\begin{aligned}
p^{2} f\left(2^{m s} q\right) & =h\left(2^{m s} p q\right)-h\left(2^{m s} p\right) q^{2} \\
& =2^{2 m s} p^{2} f(q)+h\left(2^{m s} p\right) q^{2}-h\left(2^{m s} p\right) q^{2} \\
& =2^{2 m s} p^{2} f(q)
\end{aligned}
$$

for all $p, q \in \mathcal{B}$, and so $p^{2} f(q)=p^{2 \frac{f\left(2^{m s} q\right)}{2^{2 m s}}}$ for all $p, q \in \mathcal{B}$ and $m \in \mathbb{N}$. Letting $m \rightarrow \infty$ yields $p^{2} f(q)=p^{2} h(q)$. Putting $p=e$, we get $h(q)=f(q)$ for all $q \in \mathcal{B}$. Hence, on $\mathcal{B}, f$ is a $*-$ quadratic derivation. 


\section{Derivations on random $C^{*}$-ternary algebras}

A complex random Banach space $\left(\mathcal{B}, \mu, T, T^{\prime}\right)$, which has a ternary product $(f, g, h) \longmapsto$ $[f, g, h]$ of $\mathcal{B}^{3}$ into $\mathcal{B}$, is a random $C^{*}$-ternary algebra if (see [29]):

(1) $[\xi f+v, g, h]=\xi[f, g, h]+[v, g, h]$ for all $\xi \in \mathbb{C}$;

(2) $[f, \xi g+v, h]=\xi[f, g, h]+[f, v, h]$ for all $\xi \in \mathbb{C}$;

(3) $[f, g, \xi h+v]=\xi[f, g, h]+[f, g, v]$ for all $\xi \in \mathbb{C}$;

(4) $[f, g,[h, k, j]]=[f,[k, h, g], j]=[[f, g, h], k, j]$;

(5) $\|[f, g, h]\| \leq\|f\| \cdot\|g\| \cdot\|h\|$;

(6) $\|[f, f, f]\|=\|f\|^{3}$;

for $f, g, h, v, k, j \in \mathcal{B}$.

If $\left(\mathcal{B}, \mu, T, T^{\prime}\right)$ has the unit $e$ satisfying $f=[f, e, e]=[e, e, f]$ for all $f \in \mathcal{B}$, then the random $C^{*}$-ternary algebra has unit $e$. If for $f \in \mathcal{B}$, we have $[e, f, e]=f^{*}$, then $*$ is an involution on the $C^{*}$-ternary algebra. A $C^{*}$-ternary derivation is a mapping $\delta: \mathcal{B} \longrightarrow \mathcal{B}$ such that

$$
\begin{aligned}
& \delta([f, g, h])=[\delta(f), g, h]+[f, \delta(g), h]+[f, g, \delta(h)], \\
& \delta(\xi f+g)=\xi \delta(f)+\delta(g)
\end{aligned}
$$

for all $f, g, h \in \mathcal{B}$ and $\xi \in \mathbb{C}$. Recall that $\delta([e, f, e])=[e, \delta(f), e]$ implies that $\delta$ is an involution.

Theorem 5.1 Assume that $\mathcal{B}$ is a random $C^{*}$-ternary algebra which has the unit e. Suppose that $\psi_{1}: \mathcal{B}^{2} \longrightarrow[0, \infty)$ and $\psi_{2}: \mathcal{B}^{3} \longrightarrow[0, \infty)$ are functions. Let $f: \mathcal{B} \longrightarrow \mathcal{B}$ be a mapping such that

$$
\begin{aligned}
& \mu_{f(\xi p+q)-\lambda f(p)-f(q)}(t) \geq \psi_{1}(p, q, t), \\
& \mu_{f([p, q, r])-[f(p), q, r]-[p, f(q), r][p, q f f(r)]}(t) \geq \psi_{2}(p, q, r, t), \\
& \mu_{f([e, q, e])-[e, f(q), e]}(t) \geq \psi_{2}(e, q, e, t)
\end{aligned}
$$

for all $\lambda \in \mathbb{C}, p, q, r \in \mathcal{B}$ and $t>0$. Assume there exist $s \in \mathbb{N}$ and $0<L<1$ such that $\psi_{1}\left(s^{i} p, s^{j} q, s^{(i+j)} L^{(i+j)} t\right)>\psi_{1}(p, q, t), \psi_{2}\left(s^{i} p, s^{j} q, s^{k} r, s^{(i+j+k)} L^{(i+j+k)} t\right)>\psi_{2}(p, q, r, t)$ for all $p, q, r \in \mathcal{B}$ and $i, j, k=0,1$. Then on $\mathcal{B}, f$ is a $*$-derivation.

Proof Put

$$
\Psi(p, t)=\prod_{j=1}^{s-1} \psi_{1}\left(j p, p, t_{j}\right)
$$

for $p \in \mathcal{B}$ and $t>0$ where $\sum_{j=1}^{s-1} t_{j}=t$. Then we have

$$
\mu_{f(s p)-s f(p)}(t) \geq \Psi(p, t)
$$

We use similar method presented in the proof of Theorem 3.1. Let $\Gamma$ be the set of all mappings $r: \mathcal{B} \longrightarrow \mathcal{B}$. Define a function $\Delta: \Gamma \times \Gamma \longrightarrow[0, \infty]$ by

$$
\Delta(\zeta, \eta)=\inf \left\{v>0: \mu_{\zeta(z)-\eta(z)}(\nu s) \geq \Psi(z, s)\right\}
$$


for $\zeta, \eta \in \Gamma, z \in \mathcal{B}$ and $t>0$. Miheț and Radu [28] proved that $(\Gamma, \Delta)$ is a complete GM space. Define a mapping $H: \Gamma \longrightarrow \Gamma$ by $H(\zeta)(z)=s^{-1} \zeta(s z)$. Now

$$
\Delta(\zeta, \eta)=v(\zeta, \eta \in \Gamma)
$$

implies that

$$
\mu_{H(\zeta)(z)-H(\eta)(z)}(t)=\mu_{\zeta(s z)-\eta(s z)}(\nu s t) \geq \Psi(s z, s t) \geq \Psi\left(z, \frac{t}{L v}\right)
$$

and for $\zeta, \eta \in \Gamma$

$$
\Delta(H(\zeta), H(\eta)) \leq L \Delta(\zeta, \eta) .
$$

Therefore $H$ on $\Gamma$ with Lipschitz constant $L$ is a strictly contractive function. From (5.4), we have

$$
\mu(H f)(z)-f(z)(t)=\mu_{s^{-1} f(s z)-f(z)}(t)=\mu_{f(s z)-s f(z)}(s t) \geq \Psi(z, s t) .
$$

So $\Delta(H(f), f) \leq 1 /|s|$. Using Theorem 2.1, we conclude that, in the set

$$
U=\{\zeta \in \Gamma: \Delta(\zeta, H(f))<\infty\},
$$

$h: \mathcal{B} \longrightarrow \mathcal{B}$ is a unique fixed point of $H$.

Now, for every $z \in \mathcal{B}$, we have

$$
h(z)=\lim _{m \rightarrow \infty} H^{m}(f(z))=\lim _{m \rightarrow \infty} s^{-m} f\left(s^{m} z\right)
$$

which implies that $h$ is a $\mathbb{C}$-linear mapping on $\mathcal{B}$. Also, we can show that $h$ has the $C^{*}$ ternary derivation property,

$$
\begin{aligned}
& \mu_{h([p, q, r])[h(p), q, r][p, h(q), r][p, q, h(r)]}(t) \\
& \quad=\lim _{n \rightarrow \infty} \mu_{f\left(s^{3 n}[p, q, r]\right)-s^{2 n}\left[f\left(s^{n} p\right), q, r\right]-s^{2 n}\left[p, f\left(s^{n} q\right), r\right]-s^{2 n}\left[p, q, f\left(s^{n} r\right)\right]}\left(s^{3 n} t\right) \\
& \quad \geq \lim _{n \rightarrow \infty} \psi_{1}\left(s^{n} p, s^{n} q, s^{n} r, s^{3 n} t\right) \geq \lim _{n \rightarrow \infty} \psi_{1}\left(p, q, r, \frac{t}{L^{3 n}}\right)=1 .
\end{aligned}
$$

So

$$
h([p, q, r])=[h(p), q, r]+[p, h(q), r]+[p, q, h(r)]
$$

for all $p, q, r \in \mathcal{B}$. Also,

$$
\begin{aligned}
\mu_{h([e, p, e])-[e, h(p), e]}(t) & =\lim _{n \rightarrow \infty} \mu_{f\left(s^{3 n}[e, p, e]\right)-s^{2 n}\left[e, f\left(s^{n} p\right), e\right]}\left(s^{3 n} t\right) \\
& \geq \lim _{n \rightarrow \infty} \psi_{1}\left(s^{n} e, s^{n} p, s^{n} e, s^{3 n} t\right) \\
& \geq \lim _{n \rightarrow \infty} L^{3 n} \psi_{1}\left(e, p, e, \frac{t}{L^{3 n}}\right) \\
& =1,
\end{aligned}
$$

which implies that, on $\mathcal{B}, h$ is a $*$-derivation. 
Now, in (5.2), we replace $q$ by $s^{n} q, r$ by $s^{n} r$ and divide by $s^{2 n}$. Letting $n \rightarrow \infty$, we get

$$
\begin{aligned}
& \lim _{n \rightarrow \infty} \mu_{s^{-2 n}}\left(f\left(\left[p, s^{n} q, s^{n} r\right]\right)-\left[f(p), s^{n} q, s^{n} r\right]-s^{n}\left[p, f\left(s^{n} q\right), r\right]-s^{n}\left[p, q, f\left(s^{n} r\right)\right]\right) \\
& \quad=\lim _{n \rightarrow \infty} \mu_{f\left(s^{2 n}[p, q, r]\right)-s^{2 n}[f(p), q, r]-s^{n}\left[p, f\left(s^{n} q\right), r\right]-s^{n}\left[p, q, f\left(s^{n} r\right)\right]}\left(s^{2 n} t\right) \\
& \quad \geq \lim _{n \rightarrow \infty} \psi_{1}\left(p, s^{n} q, s^{n} r, s^{2 n}\right) \geq \lim _{n \rightarrow \infty} \psi_{1}\left(p, q, r \frac{t}{L^{2 n}}\right)=1,
\end{aligned}
$$

which implies that

$$
h([p, q, r])=[f(p), q, r]+[p, h(q), r]+[p, q, h(r)]
$$

for all $p, q, r \in \mathcal{B}$. Putting $f(p)-h(p)$ instead of $q$ and $r$ in (5.7) and (5.8), we obtain $\mu_{h(p)-f(p)}(t)=1$. Hence, on $\mathcal{B}, f$ is a $*$-derivation.

\section{Funding}

No funding was received.

\section{Competing interests}

The authors declare that they have no competing interests.

\section{Authors' contributions}

All authors conceived of the study, participated in its design and coordination, drafted the manuscript, participated in the sequence alignment, and read and approved the final manuscript.

\section{Author details}

${ }^{1}$ Department of Mathematics, Iran University of Science and Technology, Tehran, Iran. ${ }^{2}$ Research Institute for Natural Sciences, Hanyang University, Seoul, Republic of Korea.

\section{Publisher's Note}

Springer Nature remains neutral with regard to jurisdictional claims in published maps and institutional affiliations.

Received: 9 August 2018 Accepted: 9 November 2018 Published online: 15 November 2018

\section{References}

1. Ulam, S.M.: A Collection of Mathematical Problems. Interscience Tracts in Pure and Applied Mathematics, vol. 8. Interscience, New York (1960)

2. Hyers, D.H.: On the stability of the linear functional equation. Proc. Natl. Acad. Sci. USA 27, 222-224 (1941)

3. Rassias, T.M.: On the stability of the linear mapping in Banach spaces. Proc. Am. Math. Soc. 72, 297-300 (1978)

4. Agarwal, R.P., Saadati, R., Salamati, A.: Approximation of the multiplicatives on random multi-normed space. J. Inequal. Appl. 2017, 204 (2017)

5. Baderi, Z., Saadati, R.: Generalized stability of Euler-Lagrange quadratic functional equation in random normed spaces under arbitrary $t$-norms. Thai J. Math. 14, 585-590 (2016)

6. Schweizer, B., Sklar, A.: Probabilistic Metric Spaces. North-Holland, New York (1983)

7. Lee, S., Saadati, R.: On stability of functional inequalities at random lattice $\phi$-normed spaces. J. Comput. Anal. Appl. $15,1403-1412(2013)$

8. Wang, Z., Saadati, R.: Approximation of additive functional equations in NA Lie $C^{*}$-algebras. Demonstr. Math. 51, 37-44 (2018)

9. Mohammad, M.V., Vaezpour, S.M., Saadati, R.: On nonlinear stability of operation preserving non-Archeimedean $\rho$-functional equations in latticetic random Banach lattice spaces. J. Math. Anal. 8, 113-123 (2017)

10. Park, C., Eshaghi Gordji, M., Saadati, R.: Random homomorphisms and random derivations in random normed algebras via fixed point method. J. Inequal. Appl. 2012, 194 (2012)

11. Bao, J., Liu, X., Wang, P.: Partially equi-integral $\phi_{0}$-stability of nonlinear differential systems. J. Math. Comput. Sci. 16 , 472-480 (2016)

12. Ardabili, J.S., Samian, Z.P.: An asymptotic stability criteria of delay differential equations on time scales. J. Math. Comput. Sci. 15, 137-145 (2015)

13. Rostamy, D., Mottaghi, E.: Convergence analysis and approximation solution for the coupled fractional convection-diffusion equations. J. Math. Comput. Sci. 16, 193-204 (2016)

14. Mohammad, M.V., Vaezpour, S.M., Saadati, R.: Nonlinear stability of $\rho$-functional equations in latticetic random Banach lattice spaces. Mathematics 6(2), Article ID 22 (2018). https://doi.org/10.3390/math6020022

15. Park, C., Anastassiou, G.A., Saadati, R., Yun, S.: Functional inequalities in fuzzy normed spaces. J. Comput. Anal. Appl. 22, 601-612 (2017) 
16. Ciepliński, K.: On a functional equation connected with bi-linear mappings and its Hyers-Ulam stability. J. Nonlinear Sci. Appl. 10, 5914-5921 (2017)

17. Choi, G., Jung, S., Lee, Y.: Approximation properties of solutions of a mean value type functional inequalities J. Nonlinear Sci. Appl. 10, 4507-4514 (2017)

18. Kim, G.H., Lee, Y.W.: Superstability of Pexiderized functional equations arising from distance measures. J. Nonlinear Sci. Appl. 9, 413-423 (2016)

19. Alshybani, S., Vaezpour, S.M., Saadati, R.: Generalized Hyers-Ulam stability of mixed type additive-quadratic functional equation in random normed spaces. J. Math. Anal. 8(5), 12-26 (2017)

20. Park, C., Shin, D., Saadati, R., Lee, J.: A fixed point approach to the fuzzy stability of an AQCQ-functional equation. Filomat 30, 1833-1851 (2016)

21. Naeem, R., Anwar, M.: Jensen type functionals and exponential convexity. J. Math. Comput. Sci. 17, 429-436 (2017)

22. Park, C., Anastassiou, G.A., Saadati, R., Yun, S.: Functional inequalities in fuzzy normed spaces. J. Comput. Anal. Appl. 22, 601-612 (2017)

23. Bratteli, O., Kishimoto, A., Robinson, D.W.: Approximately inner derivations. Math. Scand. 103, 141-160 (2008)

24. Baker, J.A., Lawrence, J., Zorzitto, F.: The stability of the equation $f(x+y)=f(x) f(y)$. Proc. Am. Math. Soc. 74, 242-246 (1979)

25. Cadariu, L., Radu, V.: Fixed points and the stability of Jensen's functional equation. J. Inequal. Pure Appl. Math. 4(1), Article ID 4 (2003)

26. Diaz, J., Margolis, B.: A fixed point theorem of the alternative for contractions on a generalized complete metric space. Bull. Am. Math. Soc. 74, 305-309 (1968)

27. Jang, S.Y.: Superstability of derivations on Banach *-algebras. Adv. Differ. Equ. 2017, 193 (2017)

28. Mihet, D., Radu, V:: On the stability of the additive Cauchy functional equation in random normed spaces. J. Math Anal. Appl. 343, 567-572 (2008)

29. Zettl, Z:: A characterization of ternary rings of operators. Adv. Math. 48, 117-143 (1983)

\section{Submit your manuscript to a SpringerOpen ${ }^{\circ}$ journal and benefit from:}

- Convenient online submission

- Rigorous peer review

- Open access: articles freely available online

- High visibility within the field

- Retaining the copyright to your article

Submit your next manuscript at $\gg$ springeropen.com 